\title{
Desempenho de frangos de corte alimentados com altos valores de balanço eletrolítico em região de clima quente
}

[Performance of broilers fed high electrolyte balance diets in hot climate region]

\author{
F.M. Vieites ${ }^{1}$, A.L. Fraga (in memoriam), C.S. Souza ${ }^{2}$, G.M. Araújo ${ }^{3}$, J.G. Vargas Júnior ${ }^{4}$, \\ R.V. Nunes ${ }^{5}$, G.S.S. Corrêa ${ }^{1}$ \\ ${ }^{1}$ Universidade Federal de Mato Grosso - UFMT \\ Av. Fernando Corrêa da Costa, n⿳0 2367 - Bairro Boa Esperança \\ 78060-900 - Cuiabá - MT \\ ${ }^{2}$ Aluna de graduação - UFMT - Rondonópolis, MT \\ ${ }^{3}$ Instituto Federal de Educação Tecnológico- IFET - Cáceres \\ ${ }^{4}$ Universidade Federal do Espírito Santo - UFES - Campus de Alegre, ES \\ ${ }^{5}$ Universidade Marechal Cândido Rondon - PR
}

\begin{abstract}
RESUMO
Determinaram-se os melhores valores de balanço eletrolítico (BE) para o desempenho de frangos de corte de um a sete, um a 14, um a 21 e um a 42 dias de idade. Utilizaram-se 720 pintos comerciais, machos, criados em galpão de alvenaria, em boxes cobertos com maravalha, alimentados com dieta basal composta por milho e farelo de soja combinada com valores de BE de 200; 250; 300; 350 e 400mEq/kg. Adotou-se o delineamento inteiramente ao acaso, com seis repetições de 24 aves para cada uma das 30 unidades experimentais. Avaliaram-se o ganho de peso, o consumo da dieta e a conversão alimentar aos sete, 14, 21 e 42 dias de idade. O melhor BE estimado para o ganho de peso aos 21 e 42 dias foi de $270 \mathrm{mEq} / \mathrm{kg}$, estimado pelo modelo quadrático de regressão. Para as demais variáveis, não houve diferenças entre os BE. Recomenda-se um valor de BE entre 270 e $300 \mathrm{mEq} / \mathrm{kG}$ para frangos de corte para as fases inicial e de crescimento em regiões de clima quente.
\end{abstract}

Palavras-chave: frango de corte, acidose metabólica, IFET-Cuiabá, eletrólito, desempenho

\begin{abstract}
A trial was carried out to determine the best electrolyte balance value (EB) for broilers performance from 1 to 7,1 to 14,1 to 21 and 1 to 42 days of age. A total of 720 one-day-old chicks, males, were reared in the floor covered with shaving woods and fed corn-soybean base diets combined with 200; 250; 300; 350 e $400 \mathrm{mEq} / \mathrm{kg}$ of EB. A completely randomized experimental design with six replicates of 24 chicks each and 36 experimental units was used. Weigh gain (WG), feed intake (FC) and feed efficiency (FE) were evaluated at 7, 14, 21 and 42 days of age. The best values of EB estimated for the WG at 21 and 42 days was $70 \mathrm{mEq} / \mathrm{kg}$, estimated by a quadratic regression model. No differences among treatments were observed for the other studied traits. The results suggested EB should be around 270 up to $300 \mathrm{mEq} / \mathrm{kg}$ in high temperature regions, for broiler chicks in inicial and growth phases.
\end{abstract}

Keywords: broiler chick, metabolic acidosis, IFET-Cuiabá, electrolyte, performance

\section{INTRODUÇÃO}

A evolução genética que ocorre na avicultura nos últimos anos resultou em aves mais produtivas, porém susceptíveis a problemas metabólicos e ao estresse calórico. Com o intuito de reduzir os problemas decorrentes do estresse calórico, várias medidas já foram adotadas, incluindo o

Recebido em 10 de março de 2010

Aceito em 21 de março de 2011

E-mail: fmvieites@yahoo.com.br

Pesquisa financiada pela Fundação de Amparo à Pesquisa do

Estado de Mato Grosso - FAPEMAT 
uso de ventiladores e nebulizadores, a manipulação de nutrientes das dietas, a utilização de antitérmicos, o manejo do arraçoamento e da água de bebida e o estabelecimento de dietas com adequado balanço eletrolítico (Souza et al., 2002; Borges et al., 2003).

O equilíbrio ácido-básico está diretamente ligado aos eletrólitos ingeridos pelas aves (Minafra et al., 2009) e é determinado pela diferença entre cátions e ânions ingeridos e excretados. O sódio $\left(\mathrm{Na}^{+}\right)$, o cloro $\left(\mathrm{Cl}^{-}\right)$e o potássio $\left(\mathrm{K}^{+}\right)$são os íons responsáveis pela manutenção da pressão osmótica, pelo equilíbrio ácido-básico e pelo metabolismo da água nos tecidos dos animais. Tais elementos devem estar em equilíbrio para o bom funcionamento do sistema enzimático e para a condução e a transmissão neural e muscular (Silva et al., 2006).

O balanço eletrolítico (BE) pode ser influenciado por condições ambientais, particularmente quando as aves são submetidas a estresse e ao calor. Altas temperaturas afetam ajustes comportamentais, fisiológicos, hormonais e moleculares. Borges et al. (2003) verificaram que frangos de corte criados em condições de altas temperaturas podem apresentar alcalose respiratória, o que provocaria, diretamente, queda de desempenho zootécnico. Dessa maneira, o fornecimento de dietas com base no conceito de BE e a adição de sais na água ou na ração são medidas que podem corrigir distorções de equilíbrio ácido-base decorrentes do estresse por calor.

A suplementação de sais nas rações ou na água de bebida é uma estratégia utilizada para aumentar a ingestão de íons específicos, por corrigir mudanças no equilíbrio ácido-básico. Tal suplementação é feita mediante inclusão de compostos alcalinos para aumentar o valor do $\mathrm{BE}$, ou por meio do incremento de compostos ácidos para reduzir o $\mathrm{BE}$, de acordo com a necessidade (Dall'Stella, 2008).

Edwards Jr. (1984) citado por Franco et al. (2004), sugeriu que o BE para máximo desempenho dos frangos de corte, nas três semanas iniciais, deve ser de aproximadamente $250 \mathrm{mEq} / \mathrm{kg}$. Murakami et al. (2001) observaram que, para o máximo crescimento de frangos de corte de 21 a 42 dias de idade, seriam necessários de 249 a 261mEq/kg de BE. Segundo OviedoRondón et al. (2000), para frangos de corte com idade entre um e sete dias, o melhor BE variou entre 250 e $252 \mathrm{mEq} / \mathrm{kg}$.

O objetivo deste estudo foi determinar os melhores valores de balanço eletrolítico de dietas de frangos de corte na fase inicial e de crescimento, em uma região de clima quente, mediante a elaboração de dietas com altos níveis de BE, considerando o desempenho apresentado pelas aves.

\section{MATERIAL E MÉTODOS}

O experimento foi realizado no mês de novembro de 2005, utilizando-se 720 pintos da marca comercial Cobb, machos. As aves foram alojadas em galpão de alvenaria, dividido em 36 boxes de $4,67 \mathrm{~m}^{2}$, e o material da cama foi a casca de arroz com $10 \mathrm{~cm}$ de altura. Adotou-se programa de luz contínuo - 24 horas de luz natural + artificial. As médias das temperaturas máxima e mínima registradas durante todo o período experimental foram de 30 e $23^{\circ} \mathrm{C}$, respectivamente, e a umidade relativa média de $79 \%$.

Durante a fase inicial, as aves foram alimentadas com uma dieta à base de milho e farelo de soja, de maneira a atender às recomendações de Rostagno et al. (2000). Nas dietas, formuladas de forma a conter $\mathrm{BE}$ de $200 \mathrm{mEq} / \mathrm{kg}$, usou-se o cloreto de amônia como fonte de cloro (Tab. 1). Os valores de BE foram calculados utilizando-se a fórmula sugerida por Mongin (1981):

$\mathrm{BE}=\left(\% \mathrm{Na}^{++} \mathrm{x} 100 / 22,990 *\right)+\left(\% \mathrm{~K}^{+} \mathrm{x}\right.$ $100 / 39,102 *)$ - (\% $\left.\mathrm{Cl}^{-} \mathrm{x} 100 / 35,453 *\right)$, em que: * é o equivalente grama de $\mathrm{Na}^{++}, \mathrm{K}^{+}$ou $\mathrm{Cl}^{-}$, respectivamente.

As aves foram distribuídas uniformemente com um dia de idade e média de peso inicial de $47 \mathrm{~g}$. Utilizou-se o delineamento experimental em blocos ao acaso (DBC), com cinco níveis de BE, seis repetições e 24 aves por unidade experimental. A dieta basal foi suplementada com $\mathrm{K}_{2} \mathrm{CO}_{3}$ e $\mathrm{NaHCO}_{3}$ em substituição ao material inerte, para obter cinco níveis do $\mathrm{BE}$ : 200; 250; 300; 350 e 400mEq/kg de BE (Tab. 2). 
Desempenho de frangos de corte...

Tabela 1. Composição da dieta basal para as fases inicial e de crescimento de frangos de corte

\begin{tabular}{|c|c|c|}
\hline Ingrediente (\%) & Dieta inicial & Dieta crescimento \\
\hline Milho & 54,454 & 59,722 \\
\hline Farelo de soja & 36,179 & 30,524 \\
\hline Óleo de soja & 3,724 & 4,291 \\
\hline Calcário & 0,977 & 0,927 \\
\hline Fosfato bicálcico & 1,825 & 1,623 \\
\hline DL - Metionina (99\%) & 0,230 & 0,209 \\
\hline L - Lisina $\mathrm{HCl}(98 \%)$ & 0,153 & 0,198 \\
\hline Sal comum & 0,456 & 0,462 \\
\hline Carbonato de potássio & - & 0,104 \\
\hline Cloreto de amônia & 0,062 & - \\
\hline Cloreto colina (60\%) & 0,080 & 0,080 \\
\hline Mistura vitamínica $^{1}$ & 0,100 & 0,100 \\
\hline Mistura mineral $^{2}$ & 0,050 & 0,050 \\
\hline Virginamicina $^{3}$ & 0,050 & 0,050 \\
\hline Anticoccidiano $^{4}$ & 0,050 & 0,050 \\
\hline Antioxidante $^{5}$ & 0,010 & 0,010 \\
\hline Areia lavada (inerte) & 1,600 & 1,600 \\
\hline Total & 100,00 & 100,00 \\
\hline \multicolumn{3}{|c|}{ Composição calculada } \\
\hline Energia metabolizável (kcal/ kg) & 3.000 & 3.100 \\
\hline Proteína bruta (\%) & 21,40 & 19,30 \\
\hline Arginina digestível (\%) & 1,336 & 1,177 \\
\hline Glicina + Serina (\%) & 2,024 & 1,812 \\
\hline Metionina + Cistina digestível (\%) & 0,807 & 0,741 \\
\hline Lisina digestível (\%) & 1,143 & 1,045 \\
\hline Treonina digestível (\%) & 0,717 & 0,643 \\
\hline Triptofano digestível (\%) & 0,242 & 0,211 \\
\hline Arginina digestível (\%) & 1,336 & 1,121 \\
\hline Cálcio (\%) & 0,960 & 0,874 \\
\hline Fósforo disponível (\%) & 0,450 & 0,406 \\
\hline Sódio (\%) & 0,222 & 0,222 \\
\hline Potássio (\%) & 0,829 & 0,799 \\
\hline Cloro (\%) & 0,386 & 0,359 \\
\hline Balanço eletrolítico (mEq/kg) & 200 & 200 \\
\hline
\end{tabular}

${ }^{1}$ Rovimix (Roche) - níveis de garantia por quilo do produto: vitamina A - 10.000.000UI; vitamina D3 - 2.000.000UI; vitamina E - 30.000UI; vitamina B1 - 2,0g; vitamina B6 - 4,0g; ác. pantotênico - 12,0g; biotina - 0,10g; vitamina K3 3,0g; ácido fólico - 1,0g; ácido nicotínico- 50,0g; vitamina B12 - 15.000mcg; selênio - 0, 25g; e veículo qsp = 1.000g.

${ }^{2}$ Roligomix (Roche) - níveis de garantia por quilo de produto: manganês 16,0g; ferro - 100,0g; zinco - 100,0g; cobre - 20,0g; cobalto - 2,0g; iodo - 2,0g; e veículo qsp = 1.000g. ${ }^{3}$ Stafac ${ }^{\circledR}-50 \%$; ${ }^{4}$ Coxistac ${ }^{\circledR}$ (salinomicina) $-12 \%$; ${ }^{5}$ Hidrox-butil-tolueno. 
Vieites et al.

Tabela 2. Valores da suplementação com $\mathrm{K}_{2} \mathrm{CO}_{3}$ e $\mathrm{NaHCO}_{3}$ para compor o balanço eletrolítico (BE) das dietas usadas para frangos de corte

\begin{tabular}{cccccc}
\hline $\mathrm{BE}(\mathrm{mEq} / \mathrm{kg})$ & Dieta basal $(\mathrm{kg})$ & Inerte $(\mathrm{kg})$ & $\mathrm{K}_{2} \mathrm{CO}_{3}(\mathrm{~kg})$ & $\mathrm{NaHCO}_{3}(\mathrm{~kg})$ & Total $(\mathrm{kg})$ \\
\hline 200 & 98,4 & 1,600 & - & - & 100 \\
250 & 98,4 & 1,213 & 0,175 & 0,212 & 100 \\
300 & 98,4 & 0,827 & 0,349 & 0,424 & 100 \\
350 & 98,4 & 0,440 & 0,524 & 0,636 & 100 \\
400 & 98,4 & 0,054 & 0,698 & 0,848 & 100 \\
\hline
\end{tabular}

$\mathrm{NaHCO}_{3}$ - peso molecular (UMA) = 84; pureza: 99\%.

$\mathrm{K}_{2} \mathrm{CO}_{3}$ - peso molecular(UMA) = 138,20; pureza: 99,0\%.

No início do estudo, as aves e as rações foram pesadas, e aos sete, 14,21 e 42 dias de idade realizou-se a avaliação de ganho de peso (GP), do consumo de ação (CR) e da conversão alimentar (CA).

Os dados obtidos foram submetidos à análise de variância, e, quando os efeitos de tratamentos foram significativos $(\mathrm{P}<0,10)$, foram efetuadas análises de regressão das variáveis em relação ao balanço eletrolítico da dieta. Os modelos de regressão foram escolhidos com base na significância dos coeficientes de regressão, e utilizou-se o programa SAS (1990) para a realização das análises estatísticas.

\section{RESULTADOS E DISCUSSÃO}

Os valores de BE afetararam o GP dos pintos de corte, contudo não houve efeito significativo sobre a CA e o CR (Tab. 3). Foi observado efeito quadrático $(\mathrm{P}<0,01)$ do $\mathrm{BE}$ sobre o $\mathrm{GP}$ aos 14 e aos 21 dias, conforme as equações $\hat{Y}=0,29631$ $+0,00075143 X-0,00000149 X^{2}\left(R^{2}=0,93\right)$ e
$\hat{\mathrm{Y}}=0,56263+0,00215 \mathrm{X}-0,00000397 \mathrm{X}^{2}$ $\left(\mathrm{R}^{2}=0,94\right)$, respectivamente. Baseado nessas equações, os melhores valores de BE estimados foram de 250 e $269 \mathrm{mEq} / \mathrm{kg}$. Tais resultados estão em conformidade com o valor de BE sugerido por Mongin (1981), mas diferem da sugestão apresentada por Borgatti et al. (2004), que, ao trabalharem com pintos de um a 21 dias de idade, submetidos ao estresse por calor, encontraram melhor ganho de peso e consumo de ração para as aves alimentadas com dietas que continham BE entre 290 e $330 \mathrm{mEq} / \mathrm{kg}$.

Os resultados sugerem que, sob condições de temperaturas elevadas, os frangos de corte melhoram o desempenho com aumento do $\mathrm{BE}$ das dietas nos limites de 250 a $300 \mathrm{mEq} / \mathrm{kg}$. Menores valores baixos de BE para a fase inicial foram verificados por Vieites et al. (2004), contudo deve-se ressaltar que esses pesquisadores trabalharam com frangos mantidos em temperaturas ambientais mais baixas que as registradas neste experimento.

Tabela 3. Desempenho de pintos de um a sete, um a 14 e um a 21 dias de idade, de acordo com o balanço eletrolítico (BE)

\begin{tabular}{|c|c|c|c|c|c|c|c|c|}
\hline Característica & 200 & 250 & 300 & 350 & 400 & Média & Efeito & CV (\%) \\
\hline \multicolumn{9}{|c|}{7 dias } \\
\hline CD (g/ave) & 139 & 143 & 140 & 138 & 140 & 140 & NS & 7,09 \\
\hline GP (g/ave) & 113 & 116 & 115 & 114 & 111 & 114 & NS & 3,29 \\
\hline $\mathrm{CA}$ & 1,22 & 1,23 & 1,22 & 1,22 & 1,27 & 1,23 & NS & 7,99 \\
\hline \multicolumn{9}{|c|}{14 dias } \\
\hline CD (g/ave) & 504 & 512 & 486 & 482 & 505 & 498 & NS & 6,66 \\
\hline GP (g/ave) & 388 & 393 & 374 & 360 & 388 & 381 & $\mathrm{Q}^{*} / \mathrm{L}^{*}$ & 3,64 \\
\hline CA & 1,22 & 1,23 & 1,22 & 1,22 & 1,27 & 1,23 & NS & 5,67 \\
\hline \multicolumn{9}{|c|}{21 dias } \\
\hline CD (g/ave) & 1261 & 1277 & 1223 & 1212 & 1246 & 1244 & NS & 4,26 \\
\hline GP (g/ave) & 850 & 858 & 819 & 790 & 833 & 830 & $\mathrm{Q}^{*}$ & 3,62 \\
\hline CA & 1,48 & 1,49 & 1,49 & 1,53 & 1,50 & 1,50 & NS & 2,87 \\
\hline
\end{tabular}

CD: consumo da dieta; GP: ganho de peso; CA: conversão alimentar.

$\mathrm{Q}^{*}$ : efeito quadrático $(\mathrm{P}<0,10)$; $\mathrm{L}^{*}$ : efeito linear $(\mathrm{P}<0,10)$; NS: efeito não significativo. 
O estresse por calor pode ocasionar a alcalose respiratória nas aves. Bacila (1980), citado por Borges et al. (2003), evidenciou que a alcalose respiratória em mamíferos provoca redução da competição entre $\mathrm{H}^{+}$e $\mathrm{K}^{+}$para excreção urinária e, portanto, aumenta a perda de $\mathrm{K}+$ na urina. $\mathrm{O}$ excesso de íons de $\mathrm{K}^{+}$compete com os ânions tampões do líquido tubular renal, impedindo a remoção do $\mathrm{H}^{+}$, e, ao ser reabsorvido, pode levar a uma acidose. Em frangos, é possível que ocorra esse mesmo mecanismo acarretando aumento na exigência de $\mathrm{K}^{+}$durante a exposição a altas temperaturas ambientais.

Conforme Lana (2000), o aumento da velocidade respiratória durante o estresse por calor eleva as perdas de água, reduzindo a sensação térmica, e, ao mesmo tempo, diminui o nível de $\mathrm{CO}_{2}$ no sangue, o que leva a um incremento do $\mathrm{pH}$. Dessa maneira, é justificável o uso de compostos alcalinos, como o $\mathrm{K}_{2} \mathrm{CO}_{3}$ e o $\mathrm{NaHCO}_{2}$, com o objetivo de elevar os níveis de bicarbonato (HCO3) ${ }^{-}$no plasma e restabelecer seus níveis normais.

Na Tab. 4, apresenta-se o desempenho de frangos de um a 42 dias de idade, de acordo com o nível de BE. Houve efeito quadrático somente sobre o GP $(\mathrm{P}<0,01)$, conforme a equação $\hat{\mathrm{Y}}=$ $1,97726+0,00675 X-0,00001234 X^{2}\left(R^{2}=\right.$ 0,97), culminando com um nível ótimo de $\mathrm{BE}$ de $269 \mathrm{mEq} / \mathrm{kg}$ e 2,900 kg de ganho de peso.

Tabela 4. Desempenho de frangos de um a 42 dias de idade de acordo com o balanço eletrolítico (BE)

\begin{tabular}{ccccccccc}
\hline Característica & 200 & 250 & 300 & 350 & 400 & Média & Efeito & $\begin{array}{c}\text { CV } \\
(\%)\end{array}$ \\
\hline \multicolumn{8}{c}{ Desempenho aos 42 dias } \\
\hline CD & 4,866 & 5,066 & 5,061 & 5,100 & 5,141 & 5,047 & NS & 4,24 \\
GP & 2,836 & 2,882 & 2,910 & 2,814 & 2,705 & 2,829 & Q $^{*}$ & $2,42^{*}$ \\
CA & 1,72 & 1,76 & 1,74 & 1,81 & 1,90 & 1,79 & NS & 5,40 \\
\hline
\end{tabular}

CD: consumo da dieta; GP: ganho de peso; CA: conversão alimentar.

$\mathrm{Q}^{*}$ : efeito quadrático $(\mathrm{P}<0,05)$; NS: efeito não significativo.

Vieites et al. (2005), ao analisarem oito níveis de BE $(0 ; 50 ; \ldots ; 350 \mathrm{mEq} / \mathrm{kg})$ e dois teores de proteína bruta (20 e 23\%), recomendaram BE na faixa de $160-190 \mathrm{mEq} / \mathrm{kg}$ para se conseguir o melhor desempenho e rendimento de cortes em frangos aos 42 dias de idade, independentemente do teor de proteína analisado. Neste caso, a adição catiônica foi realizada apenas com a suplementação de uma fonte de potássio, ao contrário do presente estudo, em que ocorreu adição de $\mathrm{K}_{2} \mathrm{CO}_{3}$ e $\mathrm{NaHCO}_{2}$.

À medida que se aumentou o valor do BE acima de $300 \mathrm{mEq} / \mathrm{kg}$, houve declínio do ganho de peso dos frangos. Uma possível explicação baseia-se na relação $\mathrm{Na} K \mathrm{~K}$, que deve ser de 0,4 para frangos em crescimento, segundo Rostagno (2000). Com a adição simultânea de quantidades iguais em $\mathrm{mEq} / \mathrm{kg}$ de $\mathrm{NaHCO}_{3}$ e de $\mathrm{K}_{2} \mathrm{CO}_{3}$, a relação Na:K aumentou. Na dieta basal utilizada neste trabalho, a relação $\mathrm{Na} K \mathrm{~K}$ foi de 0,28 , enquanto nas rações com BE de $400 \mathrm{mEq} / \mathrm{kg}$, a relação $\mathrm{Na}: \mathrm{K}$ foi de 0,71 , e o teor de potássio chegou a 1,5\%. Quando há excesso de potássio, o organismo deixa de eliminar prótons para eliminar o cátion $\mathrm{K}^{+}$, o que levaria o organismo à alcalose. A resposta fisiológica poderia ser a inibição do consumo, a qual afetaria, consequentemente, o desempenho das aves (Vieites, 2004).

De acordo com Borges et al. (2007), o $\mathrm{K}^{+}$está envolvido em muitos processos metabólicos, incluindo o antagonismo arginina-lisina, a condução nervosa, a excitação, a contração muscular, a síntese de proteínas teciduais, a manutenção da homeostasia intracelular, as reações enzimáticas, o balanço osmótico e o equilíbrio ácido-base. Mudanças na homeostase de $\mathrm{K}^{+}$podem afetar as funções celulares e, consequentemente, as respostas produtivas das aves. Esses autores afirmaram que balanços eletrolíticos elevados - 340 e 360mEq/kg podem resultar em alcalose metabólica que interfere negativamente nas respostas produtivas.

Provavelmente, a alta temperatura registrada durante o período experimental desencadeou estresse por calor nas aves. Silva et al. (1994) relataram que altas temperaturas, além de espoliarem grande quantidade de ácido orgânico, também estão associadas às perdas de eletrólitos 
através das membranas celulares. Um dos sais utilizados na produção avícola na tentativa de minimizar as perdas do estresse por altas temperaturas é o $\mathrm{NaHCO}_{3}$.

O melhor valor de BE obtido para o GP aos 42 dias foi de $269 \mathrm{mEq} / \mathrm{kg}$, um pouco acima dos obtidos por Vieites et al. (2005), de 179 a $185 \mathrm{mEq} / \mathrm{kg}$, para a mesma característica, ao trabalharem com dois níveis proteicos. Tal diferença pode estar associada à temperatura ambiental mais elevada na região sul do estado de Mato Grosso e ao fato de a adição simultânea nas dietas experimentais, de $\mathrm{NaHCO}_{3}$ e de $\mathrm{K}_{2} \mathrm{CO}_{3}$, em quantidades iguais de $\mathrm{mEq} / \mathrm{kg}$ ter sido feita com o intuito de aumentar o BE. Dessa forma, procurou-se minimizar no organismo das aves o gasto de ATP com a bomba de sódio e de potássio, ao se aumentar a disponibilidade simultânea desses elementos nas dietas, diminuindo os efeitos deletérios da concentração excessiva de um cátion apenas.

\section{CONCLUSÕES}

Recomenda-se, para a região sul do estado de Mato Grosso, BE entre 270 e $300 \mathrm{mEq} / \mathrm{kg}$, baseado nos ganhos de peso das fases inicial e final de crescimento. A adição simultânea de fontes de sódio e potássio é mais adequada para a obtenção do melhor valor de BE para as características estudadas.

\section{AGRADECIMENTO}

Ao Instituto Federal de Educação Tecnológica de Cuiabá, pela disponibilidade de instalações para a execução do experimento e pelo incentivo ao Programa de Desenvolvimento Científico e Regional. Ao Conselho Nacional de Desenvolvimento Científico e Tecnológico, pela concessão da bolsa de estudos. À Fundação de Apoio à Pesquisa do Estado de Mato Grosso, por financiar insumos para a execução do experimento.

\section{REFERÊNCIAS BIBLIOGRÁFICAS}

BORGATTI , L.M.O.; ALBUQUERQUE, R.; MEISTER N.C. et al. Performance of broilers fed diets with different dietary electrolyte balance under summer conditions. Rev. Bras. Cienc. Avic., v.6, p.153-157, 2004.
BORGES, S.A.; MAIORKA, A.; SILVA, A.V.F. Fisiologia do estresse calórico e a utilização de eletrólitos em frangos de corte. Cienc. Rural, v.33, p.975-981, 2003.

BORGES, S.A.; SILVA, A.V.F. MAIORKA, A. Acid-base balance in broilers. World's Poultr. Sci. J., v. 63, p.73-81, 2007.

DALL'STELLA, R. Balanço eletrolítico $e$ relações de aminoácidos sulfurados e lisina digestíveis para frangos de corte. 2008. 76f. Dissertação - Universidade Federal do Paraná, Curitiba, PR.

FRANCO, J.R.G.; MURAKAMI, A.E.; SAKAMOTO, M.I. et al. Efeito dos ionóforos e do balanço eletrolítico da dieta sobre o desempenho e a incidência de discondroplasia tibial em frangos de corte na fase inicial. Rev. Bras. Zootec., v.33, p.135-145, 2004.

LANA, G.R.Q. Avicultura. Recife/PE: Rural, 2000. 268 p.

MINAFRA, C.S.; MORAES, H.K.; LOPES, A.C.C. et al. O Balanço eletrolítico e proteico dietéticos sobre as aminotranferases hepáticas, renais e séricas e teores séricos de magnésio e cloro de frangos de corte. Cienc. Anim. Bras., v.10, p.425-437, 2009.

MONGIN, P. Recent advances in dietary anioncation balance: application in poultry. Proc. Nutr. Soc., v.40, p.285-294, 1981.

MURAKAMI, A.E.; OVIEDO-RONDÓN, E.O.; MARTINS, E.N. et al. Sodium and chloride requirements of growing broiler chickens (twenty-one to forty-two days of age) fed cornsoybean diets. Poult. Sci., v.80, p.289-294, 2001.

OVIEDO-RONDON, E.O.; MURAKAMI, A.E.; FURLAN, A.C. et al. Exigências nutricionais de sódio e cloro e estimativa do melhor balanço eletrolítico da ração para frangos de corte na fase pré-inicial (1-7 dias de idade). Rev. Bras. Zootec., v.29, p.1162-1166, 2000.

ROSTAGNO, H.S. Tabelas brasileiras para aves e suínos: composição de alimentos e exigências nutricionais. Viçosa: UFV, 2000. $141 p$ 
SILVA, A.V.F.; FLEMMING, J.S.; FRANCO, S.G. Utilização de diferentes sais na prevenção do estresse calórico de frangos de corte criados em clima quente. Rev. Cienc. Agr., v. 13, p.287292, 1994.

SILVA, J.D.B.; FUENTES, M.F.F.; FREITAS, E.R. et al. Níveis de sódio em rações de pintos de corte na fase inicial. Rev. Cienc. Agron., v.37, p.84-90, 2006.

SOUZA, B.B.; BERTECHINI, A.G.; TEIXEIRA, A.S. et al. Efeito da suplementação de cloreto de potássio na dieta sobre o equilíbrio ácido-básico e o desempenho de frangos de corte no verão. Cienc. Agrotec., v.26, p.1297-1304, 2002.
VIEITES, F.M.; MORAES, G.H.K; ALBINO, L.F.T. et al. Balanço eletrolítico e níveis de proteína bruta sobre o desempenho de pintos de corte de 1 a 21 dias de idade. Rev. Bras. Zootec., v.33, p.2076-2085, 2004.

VIEITES, F.M. Balanço eletrolítico e metabolismo animal. Cad. Tecn. Vet. Zootec., n.46, p.33-39, 2004.

VIEITES, F.M.; MORAES, G.H.K.; ALBINO, L.F.T. et al. Balanço eletrolítico e níveis de proteína bruta sobre o desempenho, o rendimento de carcaça e a umidade da cama de frangos de corte de 1 a 42 dias de Idade. Rev. Bras. Zootec., v.34, p.1990-1999, 2005. 